JOURNAL DE PHYSIQUE IV

Colloque C2, supplément au Journal de Physique III, Volume 5, février 1995

\title{
Invar Behavior and Martensitic Transitions in FeNiPt Alloys
}

\author{
U. Kirschbaum, H. Zähres and E.F. Wassermann \\ Gerhard-Mercator Universität, Tieftemperaturphysik, Lotharstr. 65, 47057 Duisburg, Germany
}

\begin{abstract}
We determined the structural and the magnetic phase diagram for the disordered, ternary system FeNiPt in the Fe-rich corner in the vicinity of the $\gamma(\mathrm{fcc})-\alpha(\mathrm{bcc})$ transition region along the quasi binary cross section $\mathrm{Fe}_{1-x}\left(\mathrm{Pt}_{50} \mathrm{Ni}_{50}\right)_{x}$ with $\mathrm{x}=0.28,0.3,0.31,0.32$ and 0.35 . All alloys are characterized by X-ray investigations, magnetization measurements $M(T, H)$ $(4.2 \mathrm{~K} \leq \mathrm{T} \leq 850 \mathrm{~K})$, as well as resistivity $\mathrm{R}(\mathrm{T})$ and thermal expansion measurements $\alpha(\mathrm{T})$ below $300 \mathrm{~K}$. We observe for $\mathrm{x} \leq 0.31$ a non reversible, $\gamma-\alpha$ martensitic phase transition. In the alloys with $\mathrm{x}>0.31$ the $\gamma(\mathrm{fcc})$ phase is stable down to $4.2 \mathrm{~K}$, and pronounced magnetovolume effects (Invar-like behavior) is observed. For the alloys close to the $\gamma$ - $\alpha$ transition, the magnetic behavior $\mathrm{M}(\mathrm{T}, \mathrm{H})$ is also discussed.
\end{abstract}

\section{INTRODUCTION}

In FeNi and disordered FePt alloys in the vicinity of the $\gamma(\mathrm{fcc})-\alpha(\mathrm{bcc})$ martensitic transition pronounced magnetovolume effects (Invar) are observed [1]. In both systems these Invar anomalies are in principle the same, as shown in band calculations of the total energy as a function of magnetic moment and volume [2]. However, the absolute values of the atomic volumes of these alloy systems differ appreciably. While FePt Invar alloys have lattice constants in the range $3.74 \AA \leq a \leq 3.8 \AA$, for FeNi Invar the lattice constants is about $3.59 \AA$. As shown recently by one of the authors [2] the absolute value of the lattice constants in fcc 3d-alloys also controlls the type of magnetic order occuring in these systems as well as their structural stability. While in the range around $a=3.59 \AA$ ferromagnetic (FM) and antiferromagnetic (AF) order in fcc alloys do occur, in the volume range of the fcc FePt systems only FM order is observed. AF systems with these atomic volumes having fcc structure do not exist. In the range between these two threshold values of atomic volume, binary systems with fcc structure down to zero temperature and FM order do not exist either. However, in certain ternary systems the critical range can be "bridged", and FM ordered alloys with stable fcc structure and respective atomic volumes be achieved. One of these system is FeNiPt, which is investigated in the Fe-rich corner in the vicinity of the fcc-bcc transition region along the quasi-binary cross sections $\mathrm{Fe}_{1-x}\left(\mathrm{Pt}_{50} \mathrm{Ni}_{50}\right)_{x}(\mathrm{x}=0.28,0.3,0.31,0.32$ and 0.35$)$ within this work. We determine the magnetic and structural phase diagram in this region of FeNiPt from X-ray investigations and measurements of the magnetization $\mathrm{M}(\mathrm{T}, \mathrm{H})$ and the resistivity. The results are discussed with respect to the relation between moment-volume instabilities (Invar behavior) and martensitic phase transitions. 


\section{EXPERIMENTAL}

The polycrystalline samples are prepared in an argon arc furnace, using pure Fe (99.98\%), Ni $(99.99 \%)$ and $\mathrm{Pt}(99.95 \%)$. For attaining compositional homogeinity, the button ingots are remelted several times and then, to get cylinders of $15 \mathrm{~mm}$ length and $9 \mathrm{~mm}$ in diameter, quenched in a water cooled Copper mould. The homogeinity of each sample, determined at different positions on the cylinders by electron microprobe analysis (EMPA), is better than \pm 0.2 at $\%$. From the ingots we prepared rods with $7 \mathrm{~mm}$ in length and $6 \mathrm{~mm}$ in diameter for thermal expansion (TE) measurements and $2 \mathrm{~mm} \times 0.75 \mathrm{~mm}$ for magnetization measurements. Resistivity measurements are done on rectangular sample pieces about $8 \mathrm{~mm} \times 2 \mathrm{~mm} \times 0.5 \mathrm{~mm}$ in size. After preparation, all samples are sealed under $200 \mathrm{mbar}$ Ar in silica tubes, annealed at $1200 \mathrm{~K}$ for 6 hours and finally quenched in water to achieve disorder. Structure and lattice constants of the samples are determined by X-ray diffraction (Co-K $\mathrm{K}_{\alpha}$-radiation). The electrical resistivity $\mathrm{R}(\mathrm{T})$ is measured in two different setups in the temperature range $4.2 \mathrm{~K} \leq \mathrm{T} \leq 1000 \mathrm{~K}$ by means of a four point technique, using He as an exchange gas. Magnetization measurements $\mathrm{M}(\mathrm{T}, \mathrm{H})$ in constant fields $\mathrm{H}$ in the range $\mathrm{T}<300 \mathrm{~K}$ are carried out in a SQUID-susceptometer. For high temperatures between $300 \mathrm{~K}$ and $850 \mathrm{~K}$ a Faraday balance is used. The thermal expansion is determined by a conventional relative-coppercapacitance-cell dilatometer with the sample thermally coupled to the cell. All measurements are taken at thermal equilibrium.

\section{RESULTS}

\section{$3.1 \mathrm{Fe}_{1-x}\left(\mathrm{Pt}_{50} \mathrm{Ni}_{50}\right)_{x}$-System}

Before temperature dependent measurements are done, we determined the crystal structure and the lattice constants at $300 \mathrm{~K}$. We observe for all samples pure fcc structure, except for the sample with $\mathrm{x}=0.28$, where we find a mixing of fcc and bcc order, the amount of bcc being about $75 \%$ of the sample volume.

Fig. 1 shows the relative length change $(\Delta \mathrm{L} / \mathrm{L})$ as a function of temperature $\mathrm{T}$ as measured in the range below room temperature (RT). Though all compositions have been measured, for the sake of clearness the results of only three samples with $\mathrm{x}=0.3,0.31$ and 0.32 are presented. For $\mathrm{x}=0.3$ a martensitic phase transition at $\mathrm{M}_{S}=265 \mathrm{~K}$ is observed, as indicated by the arrow in the figure. The corresponding increase of the volume between $\mathrm{M}_{S}$ and $\mathrm{M}_{f}=200 \mathrm{~K}$, given by three times the length change, is about $1.1 \%$. Decrease of $\mathrm{T}$ down to $4.2 \mathrm{~K}$ and subsequent heating results in the large hysteresis, commonly accompanying a martensitic transition. For the sample with $\mathrm{x}=0.31$ we observe similar features, with a martensitic transformation at $\mathrm{M}_{S}=124 \mathrm{~K}$, and a volume change of about $0.32 \%$. The amount of martensite, determined by the ratio of the intensity of the (110)-bcc and (111)-fcc peak through X-ray diffraction (results not shown here), is estimated to be $25 \%$ for $\mathrm{x}=0.31$ and $65 \%$ for $\mathrm{x}=0.3$, respectively .

Lowering of the Fe-concentration by another percent, the martensitic tranformation disappears, as shown for $\mathrm{x}=0.32$ in Fig. 1 (note the change of scale by a factor of 5 for this alloy in the figure). Starting at RT, we observe a very pronounced magnetovolume effect (MVE) in this sample, supported by the observations that a) the sample expands with decreasing temperature up to a maximum at $225 \mathrm{~K}$ and $\mathbf{b}$ ) that there is nearly no difference in volume between $\mathrm{RT}$ and $4.2 \mathrm{~K}$ (Invar behavior). A measure for the size of the Invar effect is the spontaneous volume magnetostriction $\omega_{S 0}$, which is estimated to be $\omega_{S 0}=1 \cdot 6 \cdot 10^{-2}$ for this alloy. This is close to the value of the classical Invar $\mathrm{Fe}_{65} \mathrm{Ni}_{35}\left(\omega_{S 0}=1.9 \cdot 10^{-2}\right)$. Magnetovolume effects (Invar-like behavior) also occur in the austenitic (fcc) range of the alloys with $\mathrm{x}=0.3$ and 0.31 , as seen by the increase of the length (volume) in the range above $\mathrm{M}_{S}$. To check whether the small hysteresis in the range around the maximum for the sample with $\mathrm{x}=0.32$ in Fig. 1 is accompanied by a lattice distortion, i.e. by a precursor effect ("pre-martensite") we carried out X-ray investigations down to 100K. No 


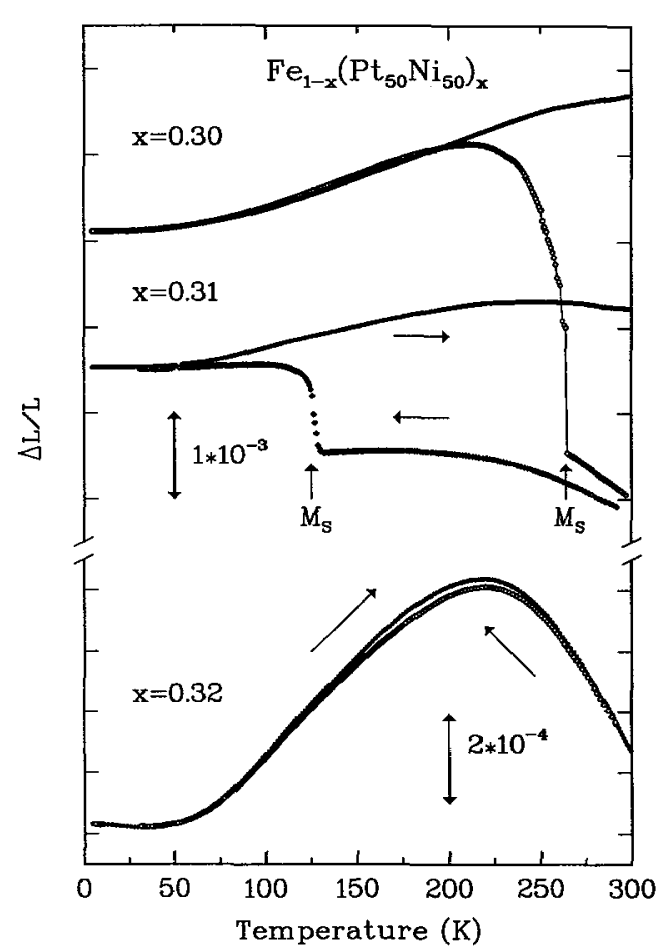

Figure 1: The relative length change $\Delta \mathrm{L} / \mathrm{L}$ of $\mathrm{Fe}_{1-x}\left(\mathrm{Pt}_{50} \mathrm{Ni}_{50}\right)_{x}$ for $\mathrm{x}=0.3,0.31$ and 0.32 versus the temperature $T$. Note the different scaling in the upper and lower part of the figure. Vertical arrows mark the martensitic transformation temperature $M_{S}$. Horizontal arrows mark the direction of measurement.
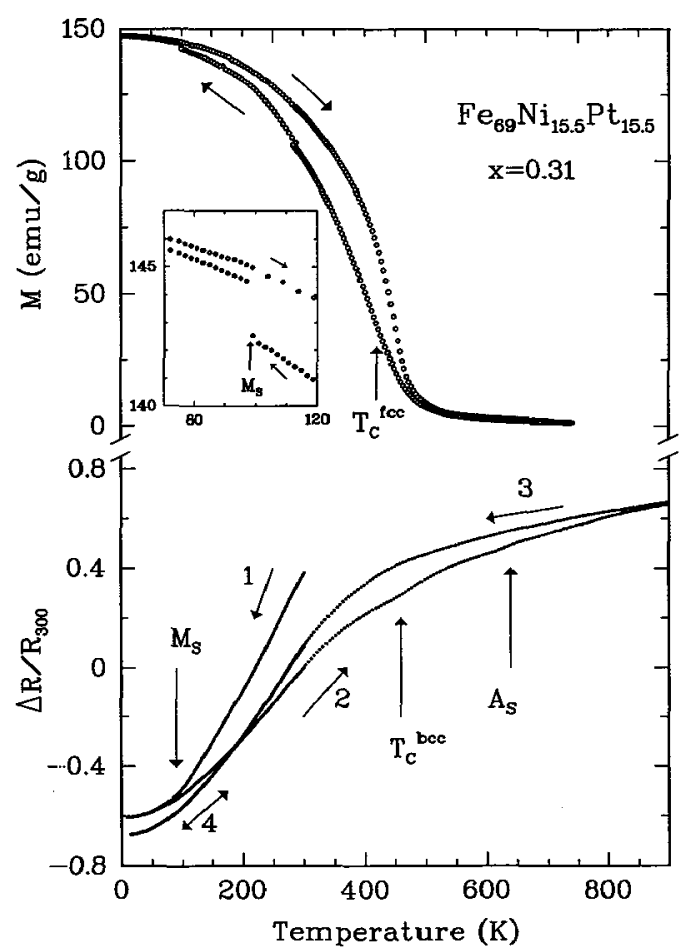

Figure 2: Magnetization $\mathrm{M}(\mathrm{T}, \mathrm{H})$ (upper part) and relative difference of the resitance normalized to $300 \mathrm{~K}, \mathrm{R}(\mathrm{T})-\mathrm{R}(300) / \mathrm{R}(300)=\Delta R / R_{300}$ in the bcc phase versus temperature for $\mathrm{Fe}_{69} \mathrm{Ni}_{15.5} \mathrm{Pt}_{15.5}$. Values and horizontal arrows mark the direction of measurement

tetragonal distortion (fct) or anomalous broadening of the linewidth could be detected. On the other hand, $\Delta \mathrm{L} / \mathrm{L}$ investigations of the sample with $\mathrm{x}=0.35$ show no hysteresis at all between cooling and heating. Thus, though the alloy with $\mathrm{x}=0.32$ lies close to the $\gamma-\alpha$ boundary, the observed precursor effect in $\Delta \mathrm{L} / \mathrm{L}$ remains unexplained.

To classify the martensitic transformation and see wether it is thermoelastic or not, we measured the magnetization $\mathrm{M}(\mathrm{T}, \mathrm{H})$ and resistivity $\mathrm{R}(\mathrm{T})$ up to higher temperatures. The results for $\mathrm{x}=0.31$ are plotted in Fig. 2. The upper part shows the saturation magnetization measured in a constant field of $\mathrm{H}=10 \mathrm{KOe}$ as a function of temperature. The arrows mark the direction of measurement with respect to $\mathrm{T}$. Starting at $300 \mathrm{~K}$, with decreasing temperature the magnetization increases (FM behavior), until at $M_{S}=98 \mathrm{~K}$ (for differences in $\mathrm{M}_{S}$, see below) we observe a sudden increase of the order of $1.3 \%$ in $\mathrm{M}(\mathrm{T})$ (see inset), since the martensite in FM ordered alloys is always FM as well. Yet, as the data reveal, the martensite obviously has a higher magnetic moment than the respective fcc phase; a result theoretically so far not understood. On further decrease of the temperature the magnetization saturates at $4.2 \mathrm{~K}$, then on reheating up to $750 \mathrm{~K}$ into the austenite range and subsequent cooling has a Curie-temperature of $T_{C}^{f c c}=420 \mathrm{~K}$. Note the occurrence of a pronounced hysteresis in the $\mathrm{M}(\mathrm{T}, \mathrm{H}=$ const $)$ behavior. The austenite start temperature $\mathrm{A}_{S}$ and the bcc Curie-temperature cannot be determined from the present cycle, because in a field of $10 \mathrm{KOe}$ the sample is paramagnetic in both the bcc and the fcc state in the high temperature 
range. Since we never observed timeholding effect, we can classify the martensitic transformation as athermal and non-thermoelastic.

To determine $A_{S}$ and $T_{c}^{b c c}$ we have carried out resistivity measurements on the same alloy. The results are given in the lower part of fig. 2 , where the relative difference of the resitance normalized to $300 \mathrm{~K}$, in the bcc phase $\mathrm{R}(\mathrm{T})-\mathrm{R}(300) / \mathrm{R}(300)=\Delta R / R_{300}$ is plotted versus the temperature. The numbers on the arrows indicate the direction of measurement. Starting at $300 \mathrm{~K}$ (1) with decreasing $\mathrm{T}$ we reach $\mathrm{M}_{S}$ at $90 \mathrm{~K}$ as indicated by the vertical arrow in the figure. After cooling down to $4.2 \mathrm{~K}$ and reheating (2) up to $1000 \mathrm{~K}$, we determine from the derivative of the resistivity (results not shown) $\mathrm{T}_{C}^{b c c}=470 \mathrm{~K}$, a value close to the temperature where the corresponding magnetization vanishes (cf. upper part of the figure), and $A_{S}=640 \mathrm{~K}$. For the other alloys similar behavior of $M(T, H)$ and $R(T)$ has been found. The critical temperatures are for $x=0.30$ and $x=0.28$ are $\mathrm{T}_{C}^{f c c}=390 \mathrm{~K}, \mathrm{~T}_{C}^{b c c}=760 \mathrm{~K}, \mathrm{~A}_{S}=635 \mathrm{~K}, \mathrm{~A}_{F}=806 \mathrm{~K}$ and $\mathrm{T}_{C}^{f c c}=330 \mathrm{~K}, \mathrm{~A}_{S}=740 \mathrm{~K}, \mathrm{~A}_{F}=840 \mathrm{~K}$ respectively. Note that after heating into the austenite range and subsequent recooling (3) and reheating (4) the discontinuous behavior in $\mathrm{R}(\mathrm{T})$ caused by the martensitic transformation vanishes. There is no more hysteresis in the resistivity and the behavior is reversible along path(4). This can be understood from the observation that slow heating to temperatures around $1000 \mathrm{~K}$ of disorderd $\mathrm{FePt}$ Invar alloys leads to (partial) structural $\mathrm{L1}_{2}$ order [3]. Ordering stabilizes the fcc phase in this system [1] and reduces $M_{S}$. Obviously, in the present experiment the slow heating rate (data point are taken in steps of $5 \mathrm{~K}$ every 10 minutes) leads to ordering in our ternary FeNiPt alloys, a result supported by the findings of Stevens et al. [3].

The results of the magnetic investigations of fcc alloys, which are stable down to $4.2 \mathrm{~K}(\mathrm{x}=0.32$ and 0.35$)$ are shown in Fig. 3, where the normalized magnetization $\mathrm{M}(\mathrm{T}, \mathrm{H}=10 \mathrm{KOe})) / \mathrm{M}(0)$, is plotted against $\mathrm{T}$ below room temperature. For comparison with a common ferromagnet, data for pure $\mathrm{Ni}$ are also plotted. One can see that with rising temperature the magnetization of the FeNiPt alloys decreases faster than that of Ni. According to the wellknown Bloch $\mathrm{T}^{3 / 2}$-law, the slope of the reduced magnetization curve is a measure of the spin wave stiffness $\mathrm{D}_{M}$ (the subscript $M$ means $D$ as determined from magnetization measurements). Thus, $\mathrm{D}_{M}$ for the presently investigated alloys is much smaller (e. g. $\mathrm{D}_{M}=94 \mathrm{meV}^{2}$ for $\mathrm{x}=0.32$ and 116 meV $\AA^{2}$ for $\mathrm{x}=0.35$, respectively) than $\mathrm{D}_{M}=433$ meV $\AA^{2}$ of pure Ni. This "magnetic weakness" is an Invar-typical feature.

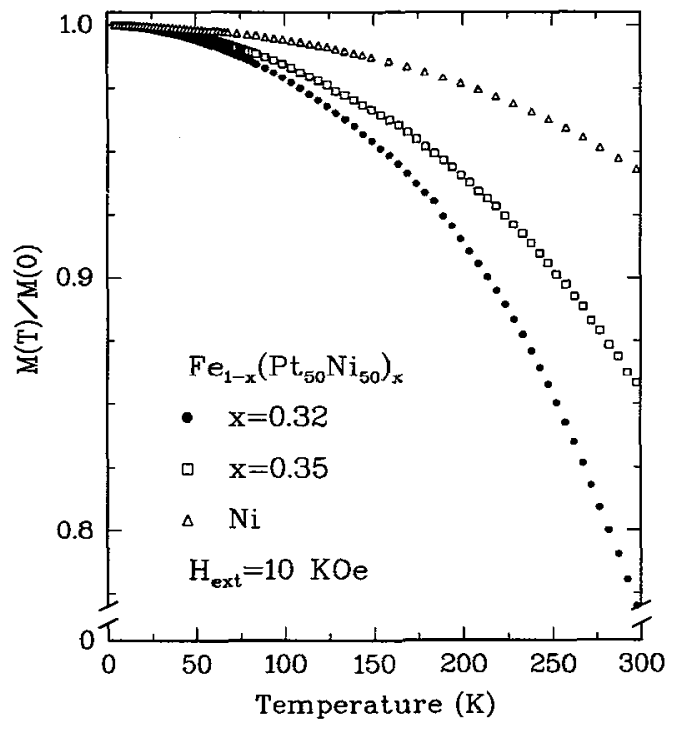

Figure 3: Normalized magnetization $\mathrm{M}(\mathrm{T}) / \mathrm{M}(0)$ versus the temperature $\mathrm{T}$ for $\mathrm{x}=0.32$ and 0.35 in comparison of pure $\mathrm{Ni}$

In Fig. 4 we collect the data determined in the present investigationas: the martensite start temperatures $\mathrm{M}_{S}$ (left hand scale, full points), the Curie temperatures $\mathrm{T}_{C}^{f c c}$ in the fcc phase (left hand scale, open squares), the lattice constants in the fcc phase at RT a $\mathrm{f}_{R T}^{f c c}$ (lower right hand scale, open triangles) and the average magnetic moments $\mu$ in units of $\mu_{B}$ per atom (upper right scale, half filled points) as a function of the composition $\mathrm{x}$ (bottom scale) and/or the electron concentration per atom e/a-ratio (top scale). One can seen that $M_{s}$ drops sharply to zero at $x=0.31$ ( $\mathrm{e} / \mathrm{a}=8.62$ ) 
characterizing a steep $\gamma-\alpha$ phase boundary. The steepness explains the uncertainties in $\mathrm{M}_{S}$ mentioned above, where different $\mathrm{M}_{S^{-}}$ values are found for samples with nominally equal composition taken from the same ingot. Small inhomogeneities and different quenching rates can cause the observed changes of $\pm 20 \mathrm{~K}$ in $\mathrm{M}_{S}$. The Curie temperatures $\mathrm{T}_{C}$ decrease almost linearly with decreasing $\mathrm{x}$ (increasing Fe-concentration) until at $\mathrm{x} \sim 0.27 \mathrm{~T}_{C}^{f c c}$, and $\mathrm{M}_{S}$ are equal. The average magnetic moments $\mu$ derived from the $\mathrm{M}(\mathrm{T}, \mathrm{H}=10 \mathrm{KOe})$ curves extrapolated to $\mathrm{T}=0 \mathrm{~K}$ are plotted in the upper part of Fig. 4 . Data of $\mu$ for the fcc alloys lie on the SlaterPauling-Curve. In the range of martensite a slight enhancement of the moment is observed. This was found in FePt Invar as well [4]. Note that there is no indication for the occurrence of antiferromagnetism (AF) in the fcc alloys as observed in the FeNi system, because we do not find a deviation from the Slater-Pauling-Curve. The reason is that the atomic volume (or the respective lattice constants $\mathrm{a}_{4.2}$ ) is too large for AF order to occur. This stems from the analysis of [2], who showed that in fcc transition metal alloys AF order only occurs when $a_{4.2}<3.64 \AA$. The lattice constants $a_{R T}^{f c c}$ are almost constant as a function of $\mathrm{x}$ in the vicinity of the $\gamma-\alpha$ boundary.

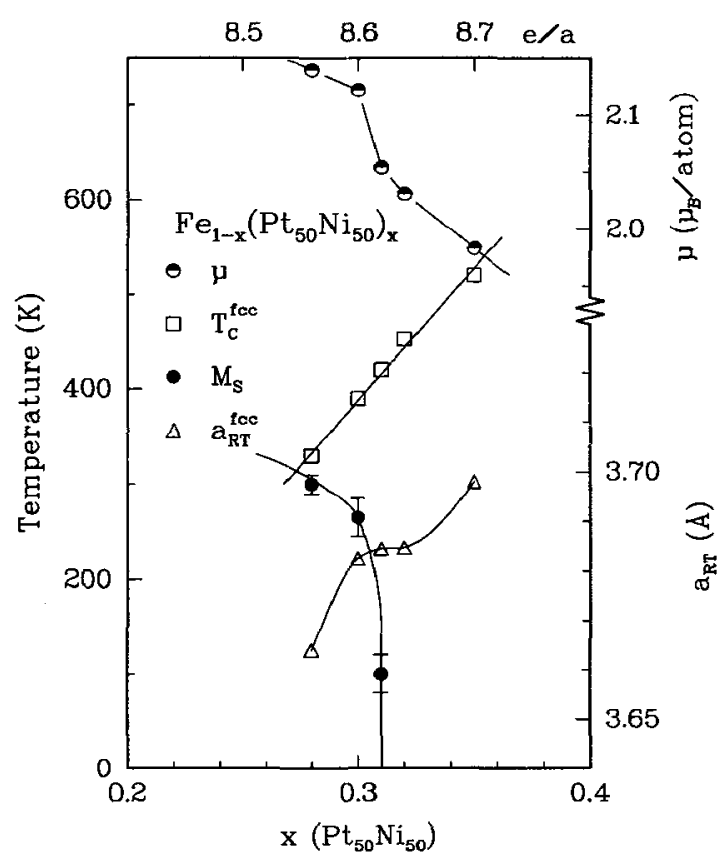

Figure 4: Phase diagram of $\mathrm{Fe}_{1-x}\left(\mathrm{Pt}_{50} \mathrm{Ni}_{50}\right)_{x}$. The average magnetic moment per atom $\mu$ (half filled points), Curie temperture in the fcc phase $T_{C}^{f c c}$ (open squares), martensite temperature $\mathrm{M}_{S}$ (full points) and lattice constant in the fcc phase at $\mathrm{T}=300 \mathrm{~K} \mathrm{a}_{R T}^{f c c}$ (open triangles) are plotted as a function of $\left(\mathrm{Pt}_{50} \mathrm{Ni}_{50}\right)$-concentration (bottom) and $\mathrm{e} / \mathrm{a}$ ratio (top), respectively.

\section{2 ternary phase diagram}

From the present investigations together with data from other authors we are able to determine part of the ternary phase diagram for disordered FeNiPt. The result is shown in Fig.5 on an enlarged scale in the Fe-rich corner up to $50 \mathrm{at} \% \mathrm{Ni}$ and $\mathrm{Pt}$. The presently investigated alloys are marked by full circles. Additional data (open circles) from our own studies of three other cross sections through this diagram (results will be presented in a future publication [5]) together with data from other authors (crosses [6], full squares [7] and full triangles [8]) are also incorporated in the diagram. Contour lines of $M_{S}=$ const. are shown by the dashed lines and those for $T_{C}=$ const. by full lines. If ternary data have not been available, data for the binary systems would have been interpolated linearly. Additionally, we show line contours of constant lattice constants at $\mathrm{T}=4.2 \mathrm{~K}$, $a_{4.2}$. Data, corrected for thermal expansion, start on the NiPt side with values reported by $[9,2]$. Note the $\gamma-\alpha$ boundary indicated by the $\mathrm{M}_{S}=0$ contour (dashed dottet line). The ranges where ordering in the ternary alloys is possible [3] are omitted for clearness. The Fe-rich corner of this diagram is of specific interest in so far as it contains both archetypical Invar systems, FeNi and $\mathrm{FePt}$ as indicated by the interval on the corresponding binary axes. The result of the present investigations is that obviously, like in the binary systems, Invar behavior and martensitic phase transition are inseperable. Both are bound to the existence of MVE. This is at least basically understood within modern band calculation [10], in which the total energy of Invar and martensite 


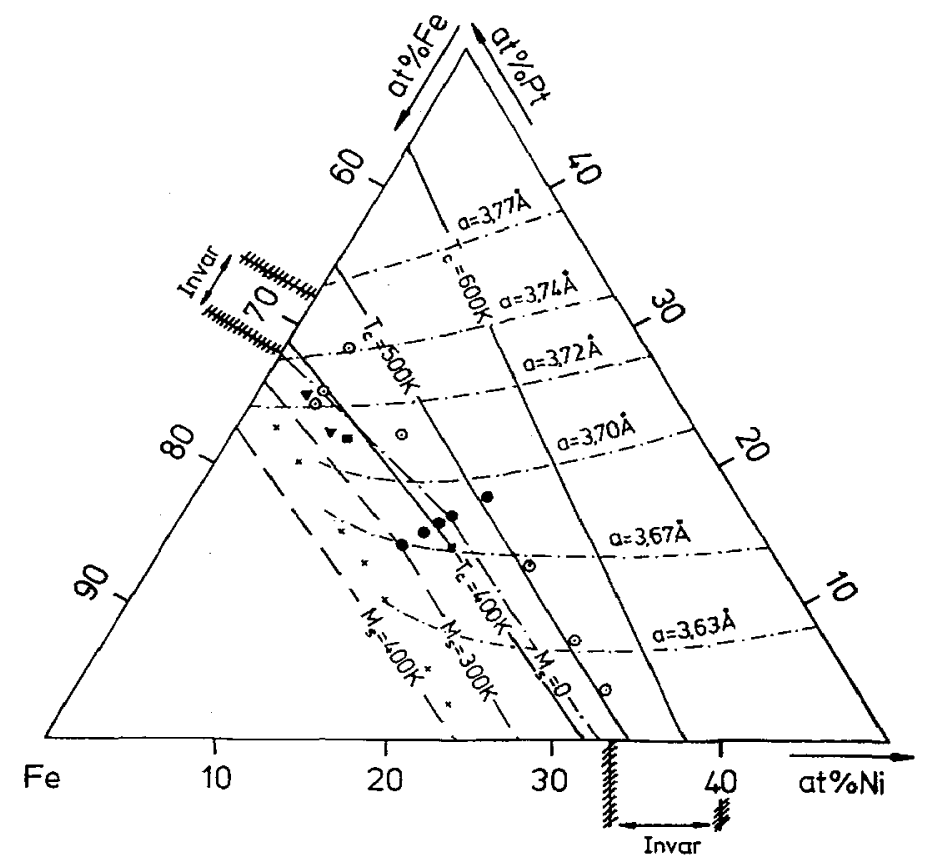

Figure 5: Ternary FeNiPt phase diagram in the Fe-rich corner. Presently investigated alloys are marked by full circles. Results from open circles will be published in future [5]. Additionally data of other authors (crosses [6], full squares [7] and full triangles [8]) are also incorporated in the diagram. Contour lines of $\mathrm{M}_{S}=$ const. are shown by the dashed lines, those for $\mathrm{T}_{C}=$ const. by full lines and those for constant lattice constants at $\mathrm{T}=4.2 \mathrm{~K}, \mathrm{a}_{4.2}$, by dashed-dotted lines.

are calculated. The key point is that the energy difference between the magnetic high spin and low spin state in Invar is of the same order of magnitude as the energy difference between the fcc and bcc phase, determined along the Bain path for the same systems.

\section{Acknowledgements}

We thank Prof. Dr. M. Acet for valuable discussions.

Work was supported by Deutsche Forschungsgemeinschaft within SFB166.

\section{References}

[1] Wassermann E.F., in Ferromagnetic Materials Vol.5 edited by K.H.J. Buschow and E.P. Wohlfarth (Elsevier, Amsterdam, 1990), pp. 237-322

[2] Wassermann E.F., J. Magn. Magn. Mater. 100 (1991) 346

3] Stevens G.T., Hatherly M. and Bowles J.S., J. Mat. Sci. 13 (1978) 499-504

[4] Sumiyama K., Shiga M. and Nakamura Y., J. Magn. Magn. Mater. 31-34 (1983) 111

5] Rellinghaus B., Kirschbaum U. and Wassermann E.F., to be published

[6] Miodownik A.P., J. Magn. Magn. Mater. 10 (1979) 126-135

[7] Kussmann A. and Jessen K., Arch. Eisenhuettenwesen 29(9) (1958) 585-594

[8] Oomi G., Iki K., Shibata K. and Aya Y., J. Magn. Magn. Mater. 104\&107 (1992) 2075-2076

[9] W.B. Pearson Handbook of Lattice Spacings and Structures of Metals and Alloys, Vol.2 (Pergamon Press, Oxford 1967)

[10] Hoffmann E., Herper H., Entel P., Mishra S.G., Mohn P. and Schwarz K. Phys. Rev. B 47 (1993) 5589-5596 